\section{Molecular Genotyping of Hepatitis A Virus, California, USA, 2017-2018}

\section{William S. Probert, Carlos Gonzalez, ${ }^{1}$ Alex Espinosa, Jill K. Hacker}

Author affiliation: California Department of Public Health, Richmond, California, USA

DOI: https://doi.org/10.3201/eid2508.181489

We implemented subgenomic and whole-genome sequencing to support the investigation of a large hepatitis A virus outbreak among persons experiencing homelessness, users of illicit drugs, or both in California, USA, during 20172018. Genotyping data helped confirm case-patients, track chains of transmission, and monitor the effectiveness of public health control measures.

$\mathrm{T}$ he United States has seen a resurgence of hepatitis A virus (HAV) infections; several states have reported outbreaks of HAV subgenotype IB, primarily among persons experiencing homelessness and users of illicit drugs (1). In California, during November 2016-May 2018, an outbreak of HAV IB infections resulted in 708 case-patients, 465 hospitalizations, and 21 deaths (1; https://www.cdph.ca.gov/ Programs/CID/DCDC/CDPH\%20Document\%20Library/ Immunization/2016-18CAOutbreakAssociatedDrugUseHomelessness.pdf). To better respond to the surge in hepatitis A cases and facilitate vaccine acquisition and distribution, California declared a public health emergency in October 2017 (2). As part of this response, the California Department of Public Health implemented molecular genotyping of HAV to support epidemiologic investigation of suspect cases.

We requested serum samples for symptomatic, HAV IgM-positive case-patients from local public health jurisdictions for genotyping. We amplified a segment of the HAV viral protein 1-amino terminus of 2B (VP1-P2B) genomic region by using nested reverse-transcription PCR and performed sequencing on 160 specimens collected during August 2017-May 2018 (3; Appendix, https://wwwnc.cdc.gov/EID/article/25/8/18-1489-App1. pdf). HAV subgenotype classification by VP1-P2B sequence yielded 48 IA-positive, 109 IB-positive, and 3 IIIA-positive specimens (Figure, panel A). We identified 19 unique HAV IA VP1-P2B sequences with an overall average genetic distance of $0.043 \mathrm{nt}$ substitutions per site. Eighteen (37.5\%) HAV IA specimens yielded sequences that matched 2 sequences (VRD_521_2016 and RIVM-HAV16-090),

${ }^{1}$ Current affiliation: Sonoma County Public Health Laboratory, Santa Rosa, California, USA. previously associated with HAV outbreaks among men who have sex with men (MSM) (4,5). All 18 case-patients were male, and all but 1 identified as MSM.

We identified 11 unique VP1-P2B sequences with an overall average genetic distance of $0.014 \mathrm{nt}$ substitutions per site among the HAV IB specimens. Phylogenetic analysis indicated 2 distinct HAV IB clusters: 1 cluster of 9 closely related sequences (CA IB cluster) identified from 107 California patients, and 1 cluster of 2 specimens matching sequences associated with a concomitant outbreak in Michigan (MI IB cluster) (1; Figure). Both case-patients with MI IB strains reported traveling to Michigan during the probable period of exposure. A search of GenBank and Hepatitis A Laboratory Network databases failed to reveal any exact matches to the CA and MI IB outbreak sequences (6; https://www.rivm.nl/en/Topics/H/HAVNET). However, Hepatitis A Laboratory Network sequence similarity analysis showed that the CA IB strains were most closely related to strains found in the Middle East and the MI IB strains to strains found in East Africa (data not shown). Three specimens with unique VP1-P2B sequences were classified as subgenotype IIIA, a genotype rarely reported in the United States (7). Two of these IIIA sequences (V17S07440 and V18S00013) shared $99.1 \%$ and $99.7 \%$ sequence identity, respectively, with strains from a 2018 outbreak in Denmark associated with dates imported from Iran (S. Midgley, Statens Serum Institut, Denmark, pers. comm., email, 2018 Aug 22). Neither of those case-patients had traveled internationally or had other known HAV risk factors within their exposure period. However, 1 case-patient had consumed dates from Iran, and the other reported eating dates from a local Middle Eastern grocery store.

We processed selected specimens representing the major IB VP1-P2B sequence variants for whole-genome sequencing (Appendix). Whole-genome sequencing of HAV can provide higher resolution strain typing than sequencing of short subgenomic regions (8). We deposited nearly complete genome sequences (7,306 nt) for strains representing the CA and MI outbreaks in GenBank (accession nos. MH577308-14). Phylogenetic comparison with other IB genome sequences in GenBank confirmed that the CA and MI IB outbreak sequences represented distinct clades (Figure, panel B). The CA IB outbreak strains shared 95.5\%-95.6\% nt and $99.7 \%$ aa sequence identity with the HAV IB reference strain, HM175. Similarly, the MI IB outbreak strains shared 95.8\% nt and 99.6\% aa sequence identity with HM175.

We rapidly implemented genotyping to help guide the public health response to a surge in reported HAV infection cases in California. Paired with epidemiologic data, genotyping information was used to confirm cases, distinguish outbreak-related cases from sporadic cases, track modes and chains of transmission, and identify populations at increased risk for infection. Our study revealed 


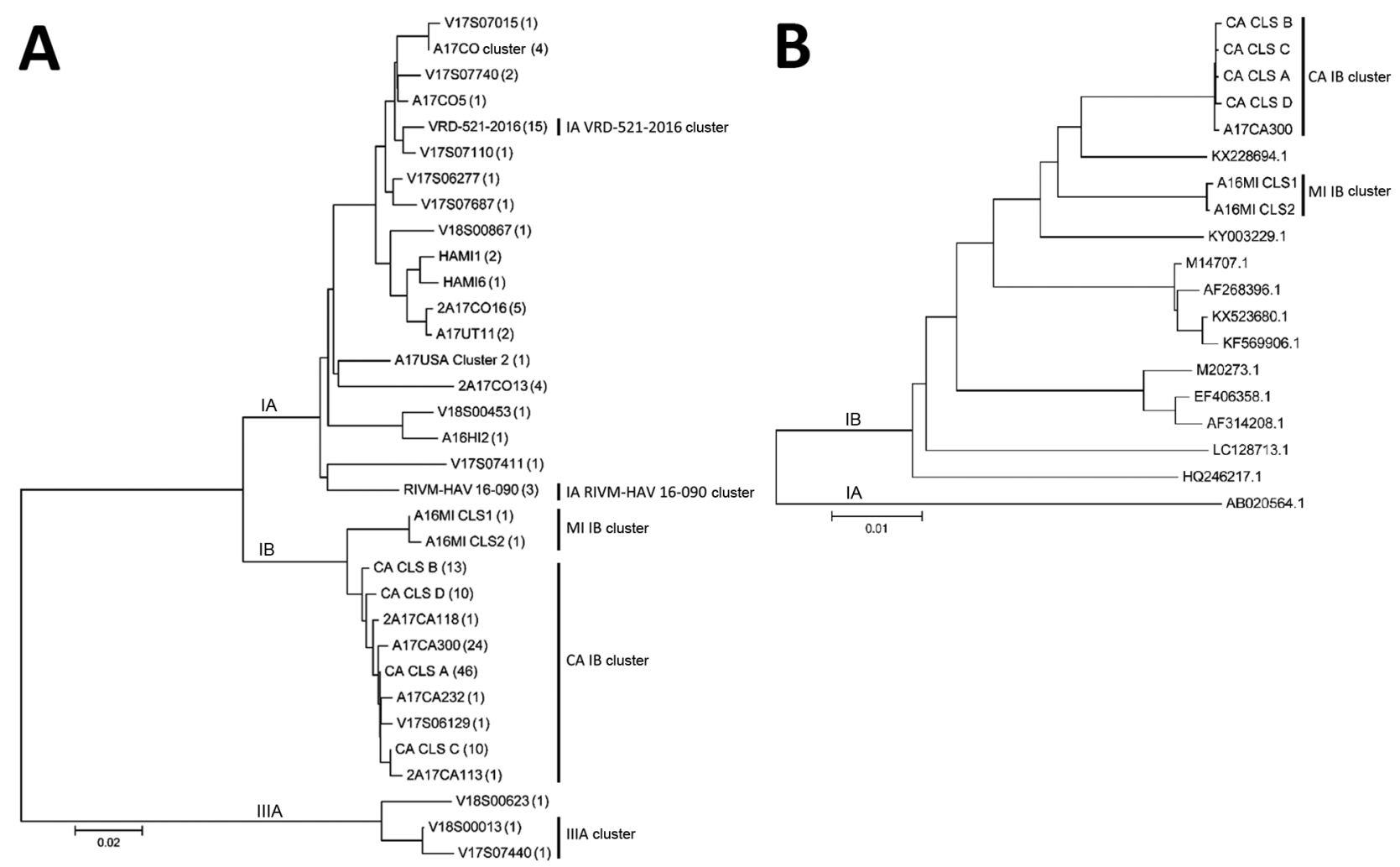

Figure. Phylogenetic analysis of HAV sequences from California, USA, and reference sequences. A) Comparison of VP1-P2B sequences obtained for 160 specimens. The number of specimens represented by each VP1-P2B sequence is indicated within parentheses. B) Comparison of nearly complete genome sequences (7,306 nt) for representative subgenotype IB cluster strains with HAV IB strain sequences found in GenBank. The genome sequence (M14707.1) represents the IB reference strain (HM175). A HAV subgenotype IA sequence (AB020564.1) was used as outlier for the analysis of the nearly complete genome sequences. Sequence alignments were performed with ClustalW (http://www.clustal.org), and the dendrograms were generated using the neighbor-joining algorithm and Kimura 2-parameter evolutionary model. Dendrogram branches corresponding to subgenotype lineage are labeled. Identified clusters of HAV are shown to the right of each dendrogram. HAV, hepatitis A virus. Scale bars indicate evolutionary distance.

several phylogenetic clusters of HAV. A large cluster of IB strains was confirmed as the primary cause of an outbreak that was chiefly transmitted person-to-person and was associated with risk factors of homelessness and illicit drug use (1). Genetically similar strains and risk factors have since been described for outbreaks in other states (1). Partly because of these outbreaks, hepatitis A vaccination was recently recommended for persons experiencing homelessness; recommendations for vaccination of users of injection and noninjection drugs were established in 1996 (9,10). By April 2018, implementation of public health control measures, including educational awareness and targeted vaccination and environmental remediation, reduced the number of reported HAV infection cases to baseline levels in California.

Limitations of our investigation were the paucity of archival genotyping data from California for strain comparisons and the lack of genotyping capabilities during early stages of the IB outbreak. Sustained public health laboratory capacity for HAV genotyping, along with diligent epidemiologic surveillance, offer the opportunity to detect outbreaks earlier and monitor the effectiveness of prevention and control efforts in California.

\section{Acknowledgments}

We gratefully acknowledge Sumathi Ramachandran and Guo-liang Xia for initially identifying the IB clusters and for their technical support in implementing VP1-P2B genotyping. We are also grateful for the epidemiological and data support provided by Monique Foster and Jennifer Zipprich, Rosie Glenn-Finer, Cynthia Yen, and Maria Salas.

\section{About the Author}

Dr. Probert is a senior microbiologist at the Viral and Rickettsial Disease Laboratory, California Department of Public Health. His research focuses on the development of molecular diagnostic tools for the detection of infectious agents. 


\section{References}

1. Foster M, Ramachandran S, Myatt K, Donovan D, Bohm S, Fiedler J, et al. Hepatitis A virus outbreaks associated with drug use and homelessness - California, Kentucky, Michigan, and Utah, 2017. MMWR Morb Mortal Wkly Rep. 2018;67:1208-10. http://dx.doi.org/10.15585/mmwr.mm6743a3

2. Office of the Governor of California. Governor Brown declares state of emergency to increase supply of hepatitis A vaccines [cited 2018 Jul 23]. https://www.ca.gov/archive/gov39/2017/10/13/ news20018/index.html

3. Hutin YJF, Pool V, Cramer EH, Nainan OV, Weth J, Williams IT, et al.; National Hepatitis A Investigation Team. A multistate, foodborne outbreak of hepatitis A. N Engl J Med. 1999;340: 595-602. http://dx.doi.org/10.1056/NEJM199902253400802

4. Beebeejaun K, Degala S, Balogun K, Simms I, Woodhall SC, Heinsbroek E, et al. Outbreak of hepatitis A associated with men who have sex with men (MSM), England, July 2016 to January 2017. Euro Surveill. 2017;22:30454. http://dx.doi.org/ 10.2807/1560-7917.ES.2017.22.5.30454

5. Freidl GS, Sonder GJ, Bovée LP, Friesema IH, van Rijckevorsel GG, Ruijs WL, et al. Hepatitis A outbreak among men who have sex with men (MSM) predominantly linked with the EuroPride, the Netherlands, July 2016 to February 2017. Euro Surveill. 2017;22:30468. http://dx.doi.org/10.2807 /1560-7917.ES.2017.22.8.30468

6. Clark K, Karsch-Mizrachi I, Lipman DJ, Ostell J, Sayers EW. GenBank. Nucleic Acids Res. 2016;44(D1):D67-72. http://dx.doi.org/10.1093/nar/gkv1276

7. Nainan OV, Armstrong GL, Han XH, Williams I, Bell BP, Margolis HS. Hepatitis a molecular epidemiology in the United States, 1996-1997: sources of infection and implications of vaccination policy. J Infect Dis. 2005;191:957-63. http://dx.doi.org/10.1086/427992

8. Vaughan G, Xia G, Forbi JC, Purdy MA, Rossi LMG, Spradling PR, et al. Genetic relatedness among hepatitis A virus strains associated with food-borne outbreaks. PLoS One. 2013;8:e74546. http://dx.doi.org/10.1371/journal.pone.0074546

9. Doshani M, Weng M, Moore KL, Romero JR, Nelson NP. Recommendations of the Advisory Committee on Immunization Practices for use of hepatitis A vaccine for persons experiencing homelessness. MMWR Morb Mortal Wkly Rep. 2019;68:153-6. http://dx.doi.org/10.15585/mmwr.mm6806a6

10. CDC. Prevention of hepatitis A through active or passive immunization: recommendations of the Advisory Committee on Immunization Practices (ACIP). MMWR Recomm Rep. 1996;45(No. RR-15):1-30.

Address for correspondence: William S. Probert, California Department of Public Health, Viral and Rickettsial Disease Laboratory, 850 Marina Bay Pkwy, Richmond, CA 94804, USA; email: will.probert@cdph.ca.gov

\section{Crimean-Congo Hemorrhagic Fever, Herat Province, Afghanistan, 2017}

\author{
Aziz-ur-Rahman Niazi, \\ Mohammad Jawed Jawad, Ahmad Amirnajad, \\ Peter A. Durr, David T. Williams
}

Author affiliations: Herat University, Herat, Afghanistan (A.-u.-R. Niazi, M.J. Jawad); Department of Public Health, Herat (A. Amirnajad); CSIRO, Australian Animal Health Laboratory, Geelong, Victoria, Australia (P.A. Durr, D.T. Williams)

DOI: https://doi.org/10.3201/eid2508.181491

We studied the clinical and epidemiologic features of an outbreak of Crimean-Congo hemorrhagic fever in Herat Province, Afghanistan. The study comprised 63 patients hospitalized in 2017 . The overall case-fatality rate was $22.2 \%$; fatal outcome was significantly associated with a negative IgM test result, longer prothrombin time, and nausea.

$\mathrm{C}$ rimean-Congo hemorrhagic fever (CCHF) is a geographically widespread tickborne disease caused by the CCHF virus (genus Orthonairovirus, family Nairoviridae). In humans, $\mathrm{CCHF}$ is associated with a case-fatality rate (CFR) of $5 \%-50 \%(1)$ and is considered a major public health threat (2).

CCHF cases were first reported in Afghanistan in 1998; no additional cases were reported until 2007 (3). During 2007-2016, the Afghanistan Ministry of Public Health documented 478 cases, of which Herat Province accounted for 263 (55.0\%) (4). In 2017, an unusual increase in CCHF cases occurred in Afghanistan, mostly in Herat Province (Appendix Figures 1, 2, https://wwwnc.cdc.gov/ EID/article/25/8/18-1491-App1.pdf). We analyzed the clinical and epidemiologic features of this outbreak.

A descriptive case series study at Herat Regional Hospital during January-December 2017 was undertaken. Clinical and epidemiologic features of all confirmed and probable CCHF cases were recorded. The Human Ethics Committee of Herat University approved the study protocol (approval \#0317).

The first recorded case in this study occurred in a 90-year-old male farmer who visited Herat Regional Hospital on May 5. Later, more patients sought care for acute febrile syndrome matching the World Health Organization CCHF case definition (5). A total of 64 patients sought care for CCHF signs and symptoms over a 6-month period, of whom 1 did not consent to hospitalization and left the hospital without medical consultation. 